Preprint typeset in JINST style - HYPER VERSION

\title{
Study of the interactions of pions in the CALICE silicon-tungsten calorimeter prototype
}

\author{
The CALICE Collaboration \\ C. Adloff, Y. Karyotakis \\ Laboratoire d'Annecy-le-Vieux de Physique des Particules, Université de Savoie, CNRS/IN2P3, \\ 9 Chemin de Bellevue BP110, F-74941 Annecy-le-Vieux Cedex, France \\ J.Repond \\ Argonne National Laboratory, 9700 S. Cass Avenue, Argonne, IL 60439-4815, USA \\ J. Yu \\ Department of Physics, SH108, University of Texas, Arlington, TX 76019, USA \\ G. Eigen \\ University of Bergen, Inst. of Physics, Allegaten 55, N-5007 Bergen, Norway
}

Y. Mikami, N. K. Watson, J. A. Wilson

University of Birmingham, School of Physics and Astronomy, Edgbaston, Birmingham B15 2TT,

$U K$

T. Goto, G. Mavromanolakis;, M. A. Thomson, D. R. Ward, W. Yan ${ }^{\dagger}$

University of Cambridge, Cavendish Laboratory, J J Thomson Avenue, CB3 OHE, UK

D. Benchekroun, A. Hoummada, Y. Khoulaki

Université Hassan II Ä̈n Chock, Faculté des sciences. B.P. 5366 Maarif, Casablanca, Morocco

J. Apostolakis, A. Ribon, V. Uzhinskiy

CERN, 1211 Genève 23, Switzerland

M. Benyamna, C. Cârloganu, F. Fehr, P. Gay

Laboratoire de Physique Corpusculaire de Clermont-Ferrand (LPC), Université Blaise Pascal, CNRS/IN2P3, 24 avenue des Landais, 63177 Aubière CEDEX, France

G. C. Blazey, D. Chakraborty, A. Dyshkant, K. Francis, D. Hedin, J. G. Lima, V. Zutshi

NICADD, Northern Illinois University, Department of Physics, DeKalb, IL 60115, USA

\section{J. -Y. Hostachy, K. Krastev, L. Morin}

Laboratoire de Physique Subatomique et de Cosmologie - Université Joseph Fourier Grenoble 1 CNRS/IN2P3 - Institut Polytechnique de Grenoble, 53, rue des Martyrs, 38026 Grenoble CEDEX,

France 
N. D’Ascenzo, U. Cornett, D. David, R. Fabbri, G. Falley, K. Gadow, E. Garutti, P. Göttlicher, T. Jung, S. Karstensen, A. -I. Lucaci-Timoce, B. Lutz, N. Meyer, V. Morgunov, M. Reinecke, F. Sefkow, P. Smirnov, A. Vargas-Trevino, N. Wattimena, O. Wendt

DESY, Notkestrasse 85, D-22603 Hamburg, Germany

\section{N. Feege, M. Groll, J. Haller, R. -D. Heuer, S. Morozov, S. Richter, J. Samson}

Univ. Hamburg, Physics Department, Institut für Experimentalphysik, Luruper Chaussee 149, 22761 Hamburg, Germany

\section{A. Kaplan, H. -Ch. Schultz-Coulon, W. Shen, A. Tadday}

University of Heidelberg, Fakultat fur Physik und Astronomie, Albert Uberle Str. 3-5 2.OG Ost, D-69120 Heidelberg, Germany

\section{B. Bilki, E. Norbeck, Y. Onel}

University of Iowa, Dept. of Physics and Astronomy, 203 Van Allen Hall, Iowa City,

IA 52242-1479, USA

\section{E. J. Kim}

Chonbuk National University, Jeonju, 561-756, South Korea

G. Kim, D-W. Kim, K. Lee, S. C. Lee

Kangnung National University, HEP/PD, Kangnung, South Korea

\section{K. Kawagoe, Y. Tamura}

Department of Physics, Kobe University, Kobe, 657-8501, Japan

P. D. Dauncey, A. -M. Magnan, H. Yilmaz, O. Zorba

Imperial College, Blackett Laboratory, Department of Physics, Prince Consort Road, London SW7 2AZ, UK

\section{Bartsch; M. Postranecky, M. Warren, M. Wing}

Department of Physics and Astronomy, University College London, Gower Street, London WC1E 6BT, UK

\section{G. Green, F. Salvatore}

Royal Holloway University of London, Dept. of Physics, Egham, Surrey TW20 OEX, UK

\section{Bedjidian, R. Kieffer, I. Laktineh}

Université de Lyon, F-69622, Lyon, France ; Université de Lyon 1, Villeurbanne ; CNRS/IN2P3, Institut de Physique Nucléaire de Lyon

\section{M.-C. Fouz}

CIEMAT, Centro de Investigaciones Energeticas, Medioambientales y Tecnologicas, Madrid. Spain

\section{S. Bailey, R. J. Barlow, M. Kelly, R. J. Thompson}

The University of Manchester, School of Physics and Astronomy, Schuster Lab, Manchester M13 9PL, UK

\section{Danilov, E. Tarkovsky}

Institute of Theoretical and Experimental Physics, B. Cheremushkinskaya ul. 25, RU-117218

Moscow, Russia 
N. Baranova, D. Karmanov, M. Korolev, M. Merkin, A. Voronin M.V.Lomonosov Moscow State University, D.V.Skobeltsyn Institute of Nuclear Physics (SINP MSU), 1/2 Leninskiye Gory, Moscow, 119991, Russia

\author{
A. Frey $\$$ S. Lư, K. Seidel, F. Simon, C. Soldner, L. Weuste \\ Max Planck Inst. für Physik, Föhringer Ring 6, D-80805 Munich, Germany
}

\begin{abstract}
J. Bonis, B. Bouquet, S. Callier, P. Cornebise, Ph. Doublet, M. Faucci Giannelli, J. Fleury, H. Li, G. Martin-Chassard, F. Richard, Ch. de la Taille, R. Poeschl, L. Raux, N. Seguin-Moreau, F. Wicek

Laboratoire de L'accélerateur Linéaire, Centre d'Orsay, Université de Paris-Sud XI, BP 34, Bâtiment 200, F-91898 Orsay CEDEX, France
\end{abstract}

M. Anduze, V. Boudry, J-C. Brient, G. Gaycken, D. Jeans, P. Mora de Freitas, G. Musat, M. Reinhard, A. Rougé, M. Ruan, J-Ch. Vanel, H. Videau

Laboratoire Leprince-Ringuet (LLR) - École Polytechnique, CNRS/IN2P3, Palaiseau, F-91128 France

K-H. Park

Pohang Accelerator Laboratory, Pohang 790-784, South Korea

\title{
J. Zacek
}

Charles University, Institute of Particle \& Nuclear Physics, V Holesovickach 2, CZ-18000

Prague 8, Czech Republic

J. Cvach, P. Gallus, M. Havranek, M. Janata, M. Marcisovsky, I. Polak, J. Popule, L. Tomasek, M. Tomasek, P. Ruzicka, P. Sicho, J. Smolik, V. Vrba, J. Zalesak

Institute of Physics, Academy of Sciences of the Czech Republic, Na Slovance 2, CZ-18221

Prague 8, Czech Republic

\section{B. Belhorma, M. Belmir}

Centre National de l'Energie, des Sciences et des Techniques Nucléaires, B.P. 1382, R.P. 10001, Rabat, Morocco

\section{S. W. Nam, I. H. Park, J. Yang}

Ewha Womans University, Dept. of Physics, Seoul 120, South Korea

\section{Jong-Seo Chai, Jong-Tae Kim, Geun-Bum Kim}

Sungkyunkwan University, 300 Cheoncheon-dong, Jangan-gu, Suwon, Gyeonggi-do 440-746,

South Korea

\section{J. Kang, Y. -J. Kwon}

Yonsei University, Dept. of Physics, 134 Sinchon-dong, Sudaemoon-gu, Seoul 120-749,

South Korea 
ABSTRACT: A prototype silicon-tungsten electromagnetic calorimeter for an ILC detector was tested in 2007 at the CERN SPS test beam. Data were collected with electron and hadron beams in the energy range 8 to $80 \mathrm{GeV}$. The analysis described here focuses on the interactions of pions in the calorimeter. One of the main objectives of the CALICE program is to validate the Monte Carlo tools available for the design of a full-sized detector. The interactions of pions in the Si-W calorimeter are therefore confronted with the predictions of various physical models implemented in the GEANT 4 simulation framework.

KEYWORDS: Detector physics: concepts, processes, methods, modelling and simulations;

Calorimeter methods; Detector modelling and simulations I (interaction of radiation with matter, interaction of photons with matter, interaction of hadrons with matter, etc.) .

*Now at CERN

${ }^{\dagger}$ Now at Dept. of Modern Physics, Univ. of Science and Technology of China, 96 Jinzhai Road, Hefei, Anhui, 230026, P. R. China

‡Now at University of Sussex, Physics and Astronomy Department, Brighton, Sussex, BN1 9QH, UK

$\S$ Now at Univ. of Göttingen

IN Now at DESY 


\section{Contents}

1. Introduction 1

2. The CALICE Si-W ECAL prototype

3. The test beam

4. Monte Carlo simulation 3

5. Selection of hadronic showers 7

6. Comparison between data and simulation 9

6.1 Total ECAL energy 9

6.2 Transverse energy profile 11

6.3 Longitudinal distribution of interaction point 13

6.4 Longitudinal energy profile 15

6.5 Physics lists under development 18

7. Summary

\section{Introduction}

The current designs of the calorimetry for detectors at the ILC are in large part driven by the demands of jet energy reconstruction. A key aim is to achieve a relative energy resolution of $\sim 30 \% / \sqrt{E / \mathrm{GeV}}$; this precision would for example permit the reconstruction of the hadronic decays of the $\mathrm{W}$ and $\mathrm{Z}$ bosons with a precision comparable with their natural widths, and would thus allow the $\mathrm{W}$ and $\mathrm{Z}$ to be distinguished in their hadronic (two-jet) decay modes.

This target for jet energy resolution is roughly a factor two better than achieved in previous detectors. The most promising way to achieve it is believed to be through the "particle flow"approach [1]. The idea is that particles of different types in jets should be reconstructed in the different parts of the detector where they can be measured most precisely: the charged particles in the tracking system, photons in the electromagnetic calorimeter (ECAL) and neutral hadrons in a combination of the ECAL and the hadronic calorimeter (HCAL). The key to this concept is to minimise confusion in the pattern recognition in the calorimeters, and to achieve this, high spatial granularity is required. The optimum design of the ILC detectors can be addressed by Monte Carlo simulation, but in order to do this, it is crucial first to validate the Monte Carlo tools against data.

A first round of beam tests was performed at DESY and CERN in summer 2006, followed by more complete tests in 2007. These tests were performed with a combined system of a silicontungsten (Si-W) ECAL, followed by a hadronic calorimeter and then a coarser tail catcher, the 
latter two both built from a scintillator-iron sandwich structure. Since the ECAL has a length $\sim 1.0 \lambda_{\text {int. }}$, it will seldom fully contain a hadronic shower. However, more than half of hadronic showers will start their development in the ECAL, so it is important to investigate the properties of hadronic showers initiated in the (mainly tungsten) material of the ECAL. The ECAL also has some advantages for the study of hadronic shower properties. For example, the interaction point can be identified with good precision. The small ratio of $X_{0} / \lambda_{\text {int. }}$ (a factor $\sim 3$ smaller for tungsten than for iron) means that the electromagnetic products of the first interaction can be expected to shower rapidly after the interaction point, and we can hope to exploit the fine granularity of the detector to separate them from the hadronic products. This means that the longitudinal shower development after the interaction point is potentially a particularly interesting quantity.

In Sect. 2 we briefly describe the main features of the Si-W calorimeter and in Sect. 3 we outline the test beam setup at CERN. Then in Sect. 7 we review the Monte Carlo models with which the data are confronted. In Sect. 5 we explain the selection of data for comparison with simulation in Sect. 6.

\section{The CALICE Si-W ECAL prototype}

The ECAL prototype used in this study is a silicon-tungsten sampling calorimeter, made of 30 readout layers [2] . The active detectors were silicon wafers segmented into a $6 \times 6$ array of diode pads, each with a size of $1 \times 1 \mathrm{~cm}^{2}$. The mechanical structure was built from tungsten sheets wrapped in carbon fibre. Between each sheet of tungsten, the structure contained alveolar slots, into which detector slabs were inserted, each consisting of a further tungsten sheet sandwiched between two layers of sensors. The prototype was constructed from three stacks, each composed of ten layers of alternating tungsten and silicon, and each stack having a different tungsten thickness: $1.4 \mathrm{~mm}$ or $0.4 X_{0}$ per layer in the first stack, $2.8 \mathrm{~mm}$ or $0.8 X_{0}$ per layer in the second stack and $4.2 \mathrm{~mm}$ or $1.2 X_{0}$ per layer in the rear stack. In terms of interaction lengths, these tungsten layers each contribute $\sim 1.4 \% \lambda_{\text {int. }}, \sim 2.7 \% \lambda_{\text {int. }}$ and $\sim 4.1 \% \lambda_{\text {int. }}$ respectively. Other materials (mainly the carbon-fibre-epoxy mechanical structure, PCBs and the silicon itself) contribute alternately $\sim 0.2 \% \lambda_{\text {int. }}$ and $\sim 1.0 \% \lambda_{\text {int. }}$ between successive samplings. The overall thickness is about $20 \mathrm{~cm}$, corresponding to $\sim 24.6 X_{0}$ or $\sim 1.0 \lambda_{\text {int. }}$ at normal incidence.

A detailed description of the prototype's hardware and of its commissioning in test beams at DESY and CERN in 2006, can be found in ref. [2], to which the reader is referred for much fuller details.

The full prototype consists of a $3 \times 3$ array of wafers (i.e. an $18 \times 18$ array of $1 \mathrm{~cm}^{2}$ pads) in each layer. The version of the prototype tested at CERN in 2007 consisted of 30 layers, of which initially the first twelve were instrumented with a $3 \times 2$ array of wafers, and the remaining eighteen were fully equipped with a $3 \times 3$ array. Later in the 2007 run six more layers were completed, leaving only the first six in a $3 \times 2$ configuration.

In offline analysis, the raw hit energies in each cell have their pedestals subtracted, and are converted from raw ADC counts into Minimum Ionising Particle equivalents (MIPs), as explained in ref. [2]. This gain correction is derived by finding the most probable energy deposition in each cell in data recorded with a high energy muon beam [2], with a typical relative precision of $\sim 0.5 \%$ per pad. 

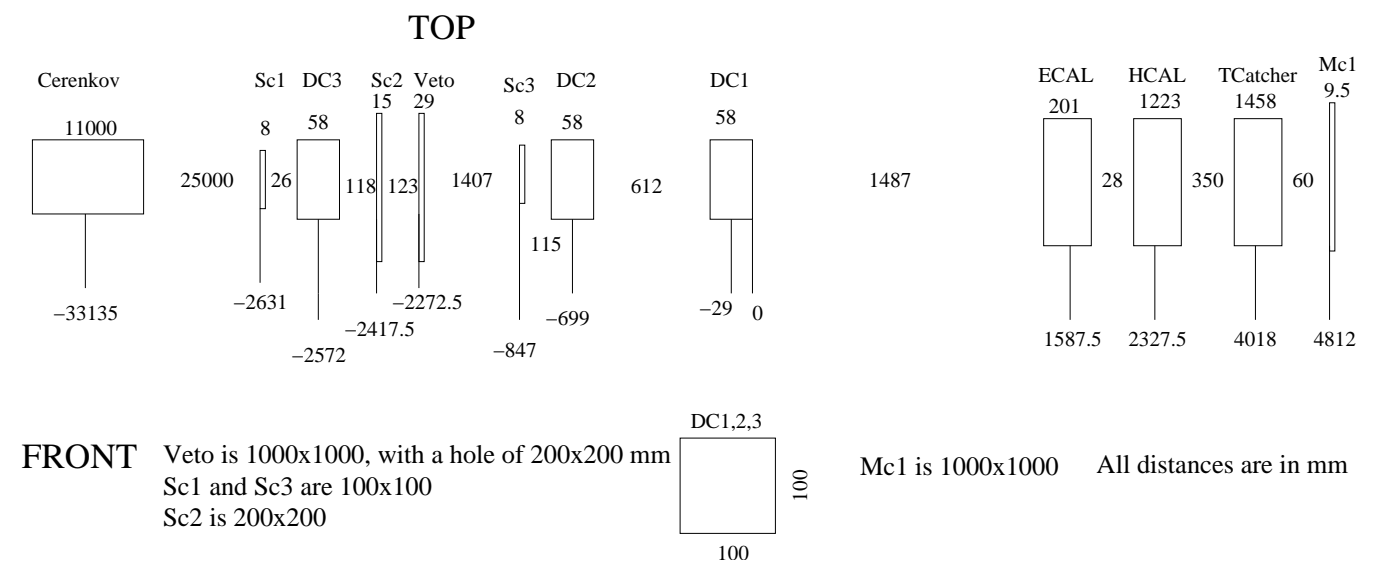

Figure 1. Schematic layout for the CALICE beam tests at CERN in 2007 (not to scale). Dimensions are indicated in $\mathrm{mm}$.

\section{The test beam}

In this paper we report on some of the data taken in 2007 in the CERN H6 test beam [3]. The layout of the CALICE calorimeters in the test beam is shown schematically in figure 11. The three calorimeters used were the Si-W ECAL, followed by an HCAL prototype [ $₫$ ] (using iron as the absorber and 38 layers of scintillator tiles with analogue readout as the active medium, using tile size $3 \times 3 \mathrm{~cm}$ in the shower core) and a tail catcher (TCMT - also an iron calorimeter with sixteen layers of $5 \mathrm{~cm}$ wide scintillator strips). The thickness of the iron sheets was $\sim 18 \mathrm{~mm}$ in the HCAL, $\sim 20 \mathrm{~mm}$ for the first eight layers of the TCMT, and $\sim 100 \mathrm{~mm}$ in the rear section of the TCMT. The ECAL and HCAL were mounted on a movable stage, providing the possibility to translate and rotate the calorimeters with respect to the beam.

Upstream of the calorimeters were scintillation counters used for triggering. A muon veto counter was also installed downstream of the TCMT. The standard trigger used for the present analysis required a coincidence between any two of the upstream counters Sc1, Sc2 and Sc3. In addition, three sets of wire chambers were operated to permit the beam position and direction to be measured upstream of the calorimeters. A Čerenkov counter was used in threshold mode to assist particle identification.

Data were recorded in 2007 using electron, hadron and muon beams with energies in the range 8 to $180 \mathrm{GeV}$. A variety of different calorimeter translations and rotations were used, and in all 200 million triggers were recorded, including calibration data. For the present study, we used data corresponding to a configuration with the beam impinging at normal incidence close to the centre of the calorimeters. The analysis uses $\pi^{-}$beams at energies of $8,10,12,15$ and $20 \mathrm{GeV}$, and $\pi^{+}$runs at 30, 50 and $80 \mathrm{GeV}$. Each run comprised typically $\sim 200,000$ triggers and $\sim 100,000$ hadronic events. For the $\pi^{-}$runs the pressure in the Čerenkov counter was set so that electrons could be vetoed, while for the $\pi^{+}$runs, it was set so as to separate protons from pions.

\section{Monte Carlo simulation}

The main purpose of the present analysis is to confront the data with Monte Carlo simulations, 
and specifically to test the different physical models available. These simulations were carried out in the framework of GEANT 4 [5], using version 4.9.3, released in December 2009. The results presented are based on samples of typically 50000 simulated events.

In order to describe the geometry of the CALICE prototypes within GEANT 4 we used the program Mokka [6], which is also capable of simulating full ILC detector geometries. For the current study, the simulation of the ECAL is the most important. Individual silicon sensors, with their pad structure and guard rings, are represented in Mokka, as well as the tungsten radiator, and other passive materials such as the carbon-fibre and epoxy of the support structure and the PCBs used for readout. The layer-by-layer staggering of the wafer positions is simulated. Similar representations of the HCAL and TCMT are also included. The detectors upstream of the calorimeter systems (Čerenkov, scintillators and tracking chambers) are also modelled by Mokka.

During particle transport in GEANT 4, Mokka records the ionisation energy deposited in sensitive detectors (Silicon pads, scintillators etc.). A simple simulation of the noise contribution in each cell is performed, as outlined in ref. [7]. At the end of the simulation, the summed energy deposits in each cell are finally converted into MIPs. The conversion factor is based on matching the minimum ionising energy peak in data and simulation. This procedure is estimated to lead to $\mathrm{a} \sim 1 \%$ systematic uncertainty in the relative energy scales of the data and simulation. As a first step in the analysis, a hit energy cut of 0.6 MIPs is imposed in both simulation and data, in order to eliminate most noise-only hits. The hits are also required to occur within $150 \mathrm{~ns}$ of the beam arrival time at the trigger counters, in order to emulate the behaviour of the readout electronics.

In the simulation, pions are simulated starting with a Gaussian transverse profile in $x$ and $y,{ }^{1}$ at a point $\sim 60 \mathrm{~m}$ upstream of the calorimeters, so that they pass through, and potentially interact in, the Črenkov counter, trigger scintillators, tracking chambers and intervening air. Hits are recorded in the scintillators and tracking chambers, and energies deposited in the scintillators are used as the basis of a simple simulation of the trigger. The beam profile in the ECAL, defined by the distributions of the shower barycentre in $x$ and $y$, is then compared between data and simulation. The input Gaussian parameters are adjusted until satisfactory agreement is achieved. A typical example of the comparison after tuning is shown in figure 2 .

GEANT 4 provides the user with a number of "physics lists" - combinations of models selected to simulate particle interactions for different species at different energies. In our study we are particularly concerned with the hadronic interactions for pions. Typically these physics lists use different models in different energy ranges, with smooth interpolation between models achieved by allowing the energy ranges to overlap, with smoothly varying random selection between the models in the changeover region. Detailed descriptions of the models can be found in ref. [8]. The following physics lists have been chosen for our investigation, based on advice and recommendations from the GEANT 4 authors:

LHEP Uses the LEP (low energy parametrised) and HEP (high energy parametrised) models, making a transition between the two over the range $25-55 \mathrm{GeV}$. These are essentially a recoding into $\mathrm{C}++$ of the GHEISHA model [9] extensively used in earlier simulations using GEANT3,

\footnotetext{
${ }^{1}$ The CALICE coordinate system is defined with $z$ along the nominal beam direction, $y$ vertical, and $x$ horizontal. The origin is at the centre of the exit face of the most downstream tracking chamber.
} 

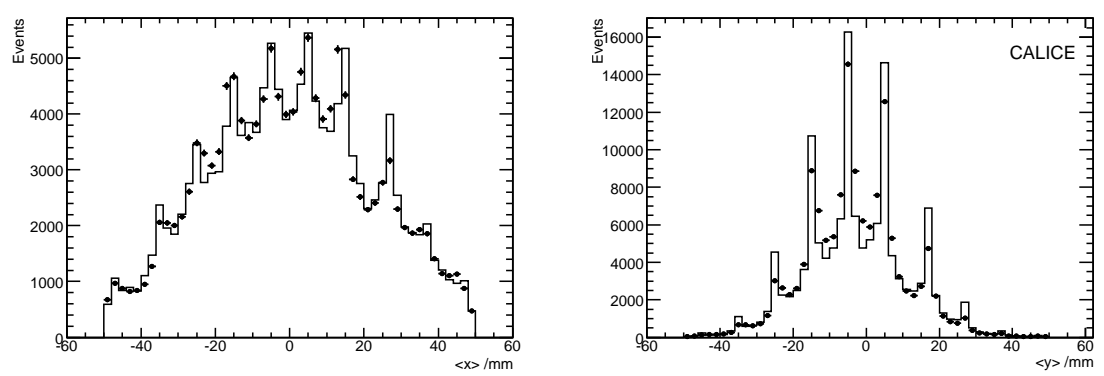

Figure 2. Example of the beam profiles in $x$ and $y$ (based on the shower centroid reconstructed in the ECAL) observed with a $20 \mathrm{GeV} \pi^{-}$beam. Data (points with error bars) are compared with the tuned simulation (solid histogram). The distributions are normalised to the same numbers of events.

for example. It is still widely used, even though it is not regarded as the state-of-the-art choice.

FTFP_BERT Uses the GEANT 4 implementation of the Bertini cascade model ${ }^{2}$ [10] for low energies, making a transition to the FTFP model, based on the GEANT 4 implementation of the Fritiof diffractive string model [11], at pion energies between 4 and $5 \mathrm{GeV}$. We have also studied the FTFP_BERT_TRV list, which has the same model content as FTFP_BERT, but with a higher transition energy, in the region 6-8 GeV. In practice, for the data presented here, we find no significant differences between these two physics lists, so we only show FTFP_BERT here.

QGSP_BERT Uses the Bertini model [10] at low energies, making a transition to the LEP (GHEISHA) model between 9.5 and $9.9 \mathrm{GeV}$, and a further transition to the QGSP model between 12 and $25 \mathrm{GeV}$. QGSP uses a GEANT 4 implementation of a string model [12] for the high energy interaction, supplemented by the GEANT 4 precompound model[13] describing deexcitation of the nucleus. We have also studied the QGSP_BERT_HP list, which has the same model content as QGSP_BERT, combined with a more accurate treatment of low energy neutron scattering. For the distributions presented in this paper we find no significant benefit in using the QGSP_BERT_HP list, and hence we only present QGSP_BERT.

QGSP_BERT_TRV has the same model content as QGSP_BERT, but with the transition between LHEP and QGSP occurring at lower energy, in the region 10-15 GeV, in order to reduce the reliance on LHEP.

QGSP_FTFP_BERT Similar to QGSP_BERT, with different transition energies, and using FTFP instead of LEP in the intermediate region.

QGS_BIC Uses the GEANT 4 binary cascade model (BIC) [14] at the lowest energies, then LEP in the intermediate region, and QGS at high energies. The BIC model is also used for the

\footnotetext{
${ }^{2}$ This model incorporates the Bertini intra-nuclear cascade model with excitons, a pre-equilibrium model, a nucleus explosion model, a fission model, and an evaporation model.
} 
rescattering of secondaries in this case (denoted QGSB below) and the GEANT 4 precompound model is used to describe deexcitation of the nucleus.

QGSP_BIC In this case the BIC model is not used for pions (only for neutrons and protons), and there is no rescattering of secondaries. LEP is used in the low energy region, and QGSP at high energies.

FTF_BIC Uses the BIC model at low energies, with a transition to Fritiof between 4 and $5 \mathrm{GeV}$. The BIC model is again used for the rescattering of secondaries in this case (denoted FTFB below) and the GEANT 4 precompound model is used to describe deexcitation of the nucleus.

The physics content of these models for pions is summarised in table 1. The QGSP_BERT physics list is generally favoured by the LHC general purpose detectors for calorimetry applications [15], based mainly on data from test beams of higher energies than those relevant for CALICE. In our analysis, we expect to be particularly sensitive to the model(s) used for the primary interaction at the energy of the incident beam. This is indicated for each physics list in table 2 .

Table 1. The table shows the physics models invoked for pion inelastic interactions in each physics list. Where ranges overlap, GEANT 4 chooses randomly between models, with probabilities varying linearly with energy over the range of overlap.

\begin{tabular}{|l||l|}
\hline Physics List & Model content (for $\left.\pi^{ \pm}\right)$ \\
\hline FTFP_BERT & Bertini $(0-5 \mathrm{GeV})$; FTFP $(>4 \mathrm{GeV})$ \\
QGSP_BERT & Bertini $(0-9.9 \mathrm{GeV})$; LEP $(9.5-25 \mathrm{GeV})$; QGSP $(>12 \mathrm{GeV})$ \\
QGSP_BERT_TRV & Bertini $(0-9.9 \mathrm{GeV})$; LEP $(9.5-15 \mathrm{GeV})$; QGSP $(>10 \mathrm{GeV})$ \\
QGSP_FTFP_BERT & Bertini $(0-8 \mathrm{GeV})$; FTFP $(6-25 \mathrm{GeV}) ;$ QGSP $(>12 \mathrm{GeV})$ \\
QGS_BIC & BIC $(0-1.3 \mathrm{GeV})$; LEP $(1.2-25 \mathrm{GeV}) ;$ QGSB $(>12 \mathrm{GeV})$ \\
QGSP_BIC & LEP $(<25 \mathrm{GeV})$; QGSP $(>12 \mathrm{GeV})$ \\
FTF_BIC & BIC $(0-5 \mathrm{GeV}) ;$ FTFB $(>4 \mathrm{GeV})$ \\
LHEP & LEP $(0-55 \mathrm{GeV})$; HEP $(>25 \mathrm{GeV})$ \\
\hline
\end{tabular}

Table 2. Indicates the physics model(s) which will be used for the primary pion interaction at each of the beam energies considered here. Where two models are indicated with a solidus, an appropriate random choice is made by GEANT 4.

\begin{tabular}{|l||c|c|c|c|c|c|c|c|}
\hline Physics List & $8 \mathrm{GeV}$ & $10 \mathrm{GeV}$ & $12 \mathrm{GeV}$ & $15 \mathrm{GeV}$ & $20 \mathrm{GeV}$ & $30 \mathrm{GeV}$ & $50 \mathrm{GeV}$ & $80 \mathrm{GeV}$ \\
\hline FTFP_BERT & FTFP & FTFP & FTFP & FTFP & FTFP & FTFP & FTFP & FTFP \\
QGSP_BERT & BERT & LEP & LEP & LEP/QGSP & LEP/QGSP & QGSP & QGSP & QGSP \\
QGSP_BERT_TRV & BERT & LEP & LEP/QGSP & QGSP & QGSP & QGSP & QGSP & QGSP \\
QGSP_FTFP_BERT & FTFP & FTFP & FTFP & FTFP/QGSP & FTFP/QGSP & QGSP & QGSP & QGSP \\
QGS_BIC & LEP & LEP & LEP & LEP/QGSB & LEP/QGSB & QGSB & QGSB & QGSB \\
QGSP_BIC & LEP & LEP & LEP & LEP/QGSP & LEP/QGSP & QGSP & QGSP & QGSP \\
FTF_BIC & FTFB & FTFB & FTFB & FTFB & FTFB & FTFB & FTFB & FTFB \\
LHEP & LEP & LEP & LEP & LEP & LEP & LEP /HEP & LEP/HEP & HEP \\
\hline \hline
\end{tabular}

GEANT 4 also provides several interesting physics lists based wholly or in part on the CHIPS model [16]. In this picture, the result of a hadronic or nuclear interaction is the creation of a quasmon (essentially an intermediate state of excited hadronic matter) which can dissipate energy by radiating particles statistically or by quark exchange with surrounding nucleons or clusters of nucleons. We have made studies of several CHIPS-based physics lists, but we choose not to present 


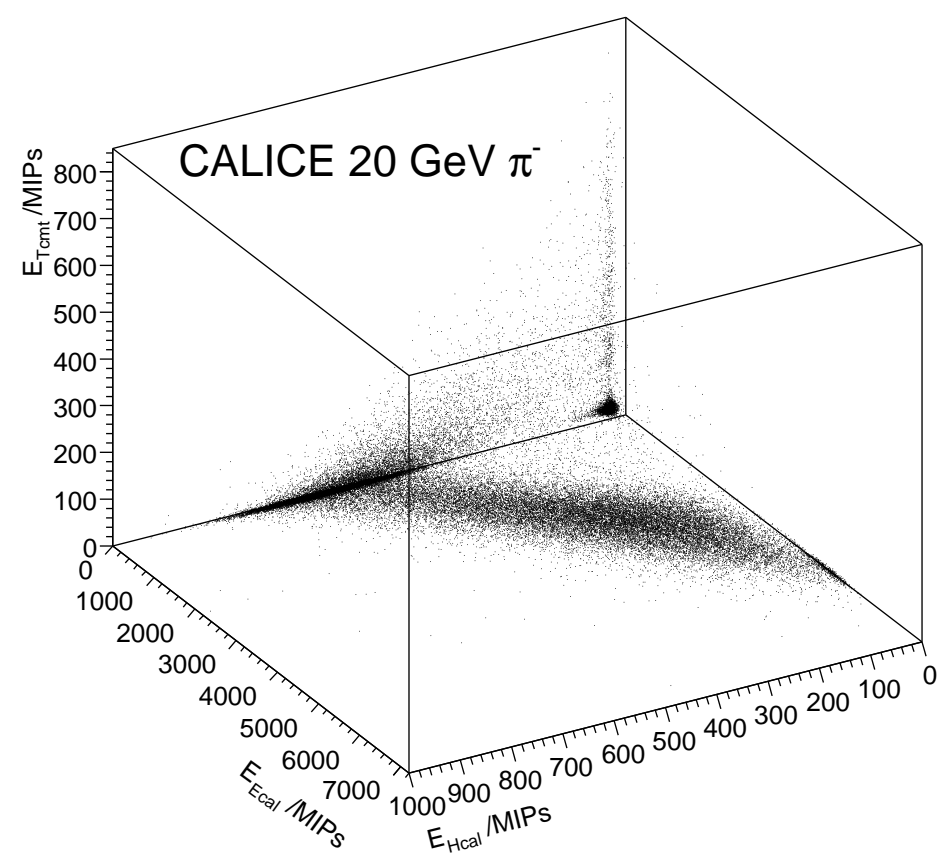

Figure 3. Showing the principle of the cuts used to remove muons and non-interacting hadrons. The energies in the three calorimeters (in MIPs) are plotted for a $20 \mathrm{GeV}$ data run. The three stacks in the ECAL are combined using weights proportional to the corresponding thicknesses of tungsten, i.e. 1:2:3.

comparisons at this stage because, as discussed in Sect. 6.5, the model is still under active development.

\section{Selection of hadronic showers}

The principal task in the selection of events is to remove muons, electrons and protons from the sample. Muons are characterised by a small energy deposition in all three calorimeters. We show in figure 3 a scatter plot of the energies seen in the three calorimeters. A clear cluster close to the origin can be ascribed to muons. Bands are also seen corresponding to sharing of energy between ECAL and HCAL, and between HCAL and TCMT, with a few showers only starting in the TCMT. Accordingly, with a $10 \mathrm{GeV}$ beam, we reject events for which the ECAL energy is less than 300 MIPs, the HCAL energy is less than 100 MIPs and the TCMT energy is less than 50 MIPs. These cuts vary linearly with energy, so that with a $30 \mathrm{GeV}$ beam, for example, they are 329,114 and 64 MIPs respectively. Pions which do not interact in the ECAL are retained in the sample, to permit comparison of the interaction cross-section between data and simulation. The fraction of events removed as muons varies with beam energy, but it is typically $\sim 5 \%$.

Double beam particles are very infrequent with the running conditions used in 2007, but in order to safely reduce any possible contribution from double beam particles, a cut is imposed, requiring that the total energy recorded in the ECAL+HCAL be less than 1.5 times the beam energy. Fewer than $0.5 \%$ of events fail this cut. In order to reduce the influence of interactions upstream 

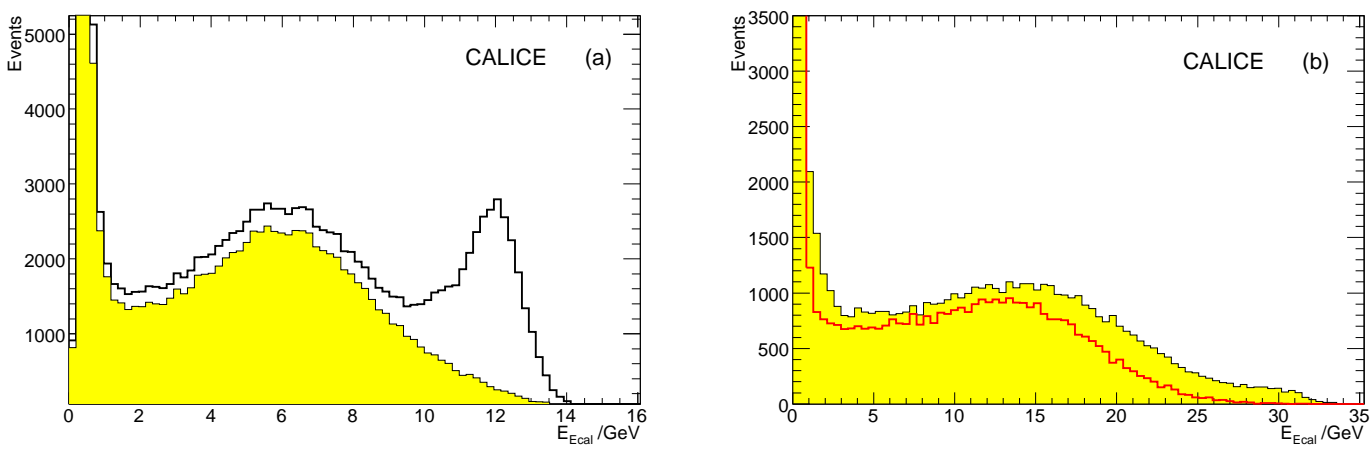

Figure 4. Energy observed in the ECAL for events with and without a signal from the beam Čerenkov counter. For this plot, the observed energy was converted from MIPS to GeV using a nominal conversion factor of $240 \mathrm{MIPs} / \mathrm{GeV}$. (a) In the case of the $12 \mathrm{GeV}$ negatively charged beam, the open region shows the total sample, and the shaded region the contribution with no Čerenkov signal. (b) For the $30 \mathrm{GeV}$ positively charged beam the shaded histogram shows triggers with a Čerenkov signal, and the open (red) histogram shows those with no Čerenkov signal.

of the calorimeters, we remove events in which more than 50 MIPs are recorded in either of the first two layers of the ECAL. This cut removes $\sim 2.5 \%$ of data, with only a weak dependence on beam energy. A cut against beam halo is also applied, requiring the shower centroid to lie within $\pm 50 \mathrm{~mm}$ in both $x$ and $y$, corresponding approximately to the trigger acceptance; this cut also serves to remove a small number of showers close to the calorimeter edge, which are especially prone to lateral leakage. This cut removes $\sim 5 \%$ of data at the lowest energy and $\sim 1 \%$ at the highest energy, where the beam is narrowest. All of the cuts described so far are applied equally to data and Monte Carlo events.

Electrons can provide a significant background to the pions in the negatively charged beams, and likewise protons are present in the higher energy positively charged beams. Their effect may be reduced by use of the Čerenkov counter. In figure Ð(a) we compare, for typical negatively and positively charged beam runs, the distribution of the energy in the ECAL for triggers with and without a Čerenkov signal. In the case of the negatively charged beam we see a clear peak at the full beam energy in the events with a Čerenkov signal, which can be ascribed to electrons depositing their full energy in the ECAL. Therefore, for the negatively charged beams we demand that the Čerenkov have a signal. Conversely, for the positively charged beams used here, the Čerenkov pressure was set so that pions (and electrons) would yield a signal, while the heavier species would not. The ECAL energy distributions with and without a Čerenkov signal are shown in figure $4(b)$; we see that for the events with no Čerenkov signal, mainly protons, the distribution is shifted to lower values, and accordingly for these runs we demand that there be no Čerenkov signal. We also note that the contribution of positrons, which would show up as a peak close to $30 \mathrm{GeV}$ in the sample of events with a Čerenkov signal, is small ${ }^{3}$.

In our previous study of electron showers in the ECAL [7], it was necessary to remove showers

\footnotetext{
${ }^{3}$ From fitting the region around the full beam energy, we estimate a positron contribution of $\sim 1 \%$ at $30 \mathrm{GeV}$, decreasing to $0.4 \%$ at $50 \mathrm{GeV}$ and $0.1 \%$ at $80 \mathrm{GeV}$. This has a negligible impact on the results shown in the present paper. No perceptible electron contribution is seen in the negatively charged beam samples.
} 

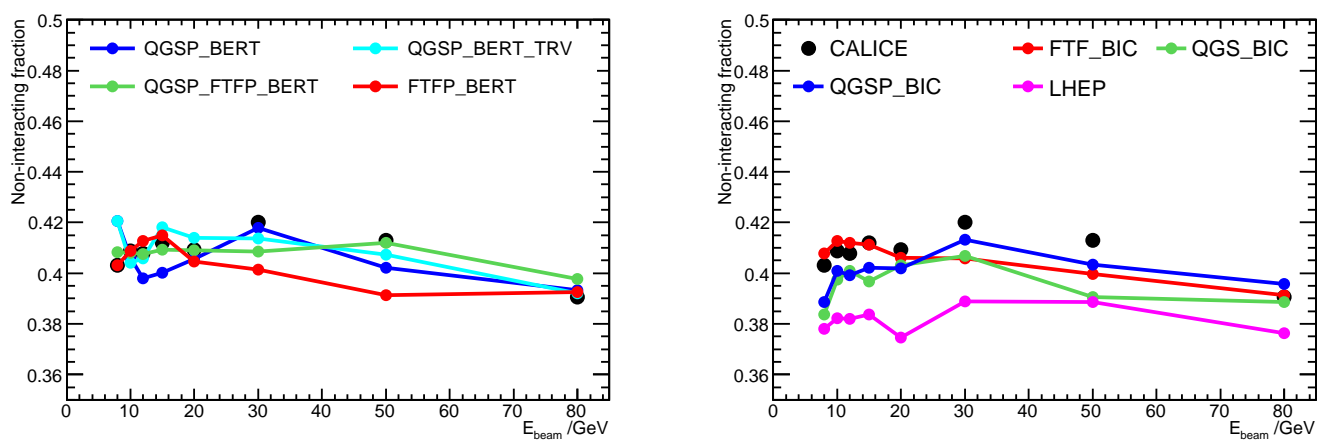

Figure 5. Mean fraction of non-interacting pions (energy $<100 \mathrm{MIPs}$ ) in the ECAL, plotted as a function of beam energy. For this purpose, the three ECAL stacks were combined with weights 1:1:1. The data are compared with the predictions of simulations using different GEANT 4 physics lists. The models are separated into two plots in the interests of clarity, with the physics lists which incorporate the Bertini model on the left, and the others on the right.

close to the edges of wafers, for two reasons. Firstly, the guard rings lead to a significant dead region ( $2 \mathrm{~mm}$ wide), which can lead to a significant fraction of the electron energy not being recorded. Secondly, we observed an effect of correlated crosstalk between the charge deposited in the guard rings and the peripheral cells of a wafer, whereby a square pattern of hits was sometimes seen in the core of a high energy shower [2]. The first of these problems is less important for hadronic showers because of their greater transverse width, and in any case the effect is sufficiently well simulated. The second problem is not seen in hadronic showers, because the energy densities in the core of the showers are much smaller. Therefore no further cuts on shower position are imposed.

\section{Comparison between data and simulation}

\subsection{Total ECAL energy}

The total energy recorded in the ECAL is a useful starting point for comparison between data and simulation. The event selection cuts were designed to retain pion events wherever they interacted in the calorimeter system. A significant fraction of pions should not start to shower in the ECAL. These events are characterised by a MIP-like energy in all layers (apart from occasional $\delta$-ray emission) and accordingly the ECAL energy shows a large peak at $\sim 50$ MIPs. The fraction of such events can be used to test the interaction cross-sections in GEANT 4. In figure 5 we show the fraction of selected pions depositing fewer than 100 MIPs in the ECAL, as a function of energy, compared with the simulations. Most of the models give a good description of the fraction of noninteracting pions at all energies, agreeing with data within 0.01-0.02. The LHEP physics list is the most discrepant. This gives confidence in the cross-sections simulated in GEANT 4. It could also be interpreted as an indication that any residual beam contamination by kaons or (anti-)protons is small, since these species would be expected to have different interaction cross-sections.

We now consider the energy deposited in the ECAL by those pions which have their first interaction in the ECAL. In the study of electromagnetic showers [7], it was appropriate to combine 

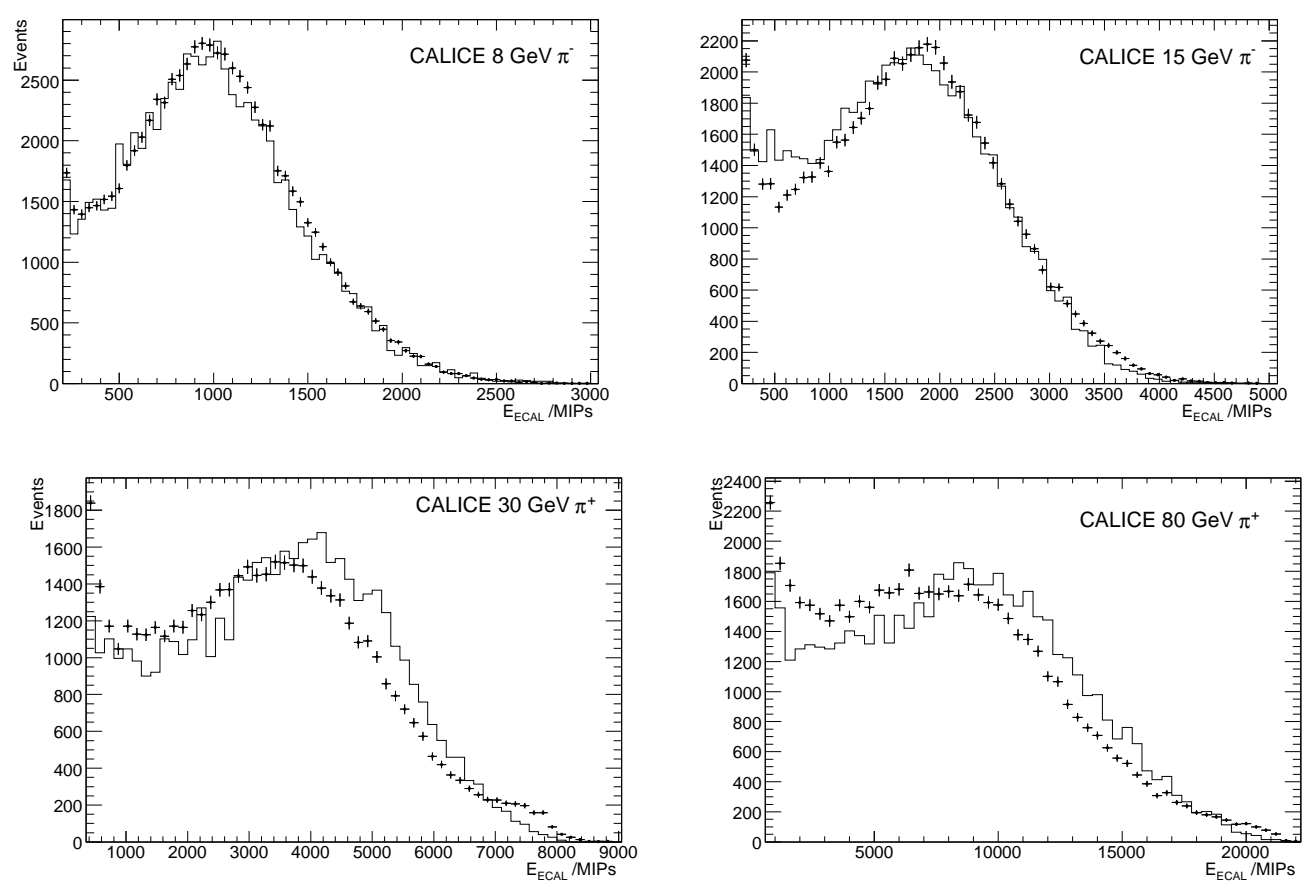

Figure 6. Distributions of total energy recorded in the ECAL at 8, 15, 30 and $80 \mathrm{GeV}$ (points with error bars), compared with Monte Carlo predictions using the QGSP_BERT physics list (solid histograms). The distributions are normalised to the same numbers of selected events (including the non-interacting peak).

the energies in the three stacks of the ECAL taking account of their relative sampling fractions. Since the thicknesses of tungsten in the three stacks lie in the ratio $1: 2: 3$, the weighting of the energy should be in roughly these ratios. It is less obvious that this is the correct procedure in the case of hadronic showers, but for the purposes of the present comparison, we choose to combine energies of each stack using the naïve weighting factors of 1,2 and 3. The level of agreement between data and simulation is not found to be sensitive to this choice. In figure 6 we compare the distribution of recorded energy in data with simulation using the QGSP_BERT physics list, at four typical energies. The non-interacting peak at low energies has been suppressed. The broad peaks in the distributions in figure 6 represent pions which started to shower in the ECAL, extending roughly to a point corresponding to all the energy being deposited in the ECAL (approximately $250 \mathrm{MIPs}$ per $\mathrm{GeV}$ ). The main peak is quite well modelled at 8 and $15 \mathrm{GeV}$, while at 30 and especially $80 \mathrm{GeV}$ the Monte Carlo predicts significantly more energy than observed in the data. ${ }^{4}$

In order to incorporate all models and energies into the analysis, a useful global measure is the average energy deposited in the ECAL, i.e. the mean values of the histograms such as those shown in figure 6, excluding the non-interacting pions. This can be computed for data and for all the GEANT 4 physics lists under consideration, and is plotted in figure 7 in the form of ratios of simulation to data. At $8 \mathrm{GeV}$, all of the models lie within $10 \%$ of the data, and most within

\footnotetext{
${ }^{4}$ In ref. [7] the energy response of the ECAL for electrons was seen to be linear up to $45 \mathrm{GeV}$. Since the energy densities in electromagnetic showers are significantly greater than those encountered in hadronic showers, we can be confident that saturation effects will not play any significant part in the hadron beam data.
} 

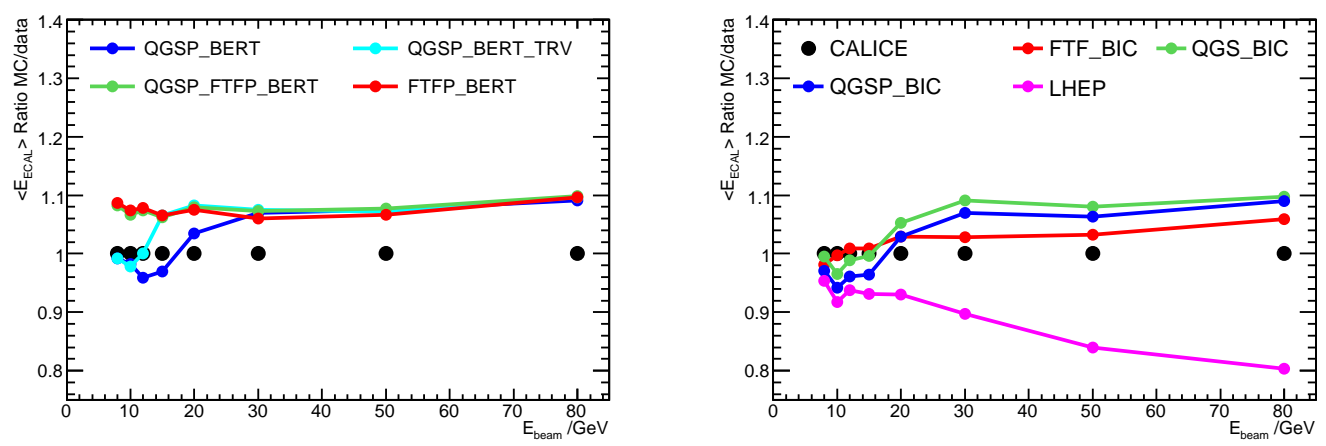

Figure 7. Ratio of simulation to data for the mean energy recorded in the ECAL, plotted as a function of beam energy. The data are compared with the predictions of simulations using different GEANT 4 physics lists.

5\%. As energy increases, the LHEP model falls steadily further below the data. The other models either remain $\sim 5-10 \%$ above the data or increase so that at high energies, all of the models lie $\sim 5-10 \%$ above the data. Overall, FTF_BIC is the most consistent with this aspect of the experimental data.

\subsection{Transverse energy profile}

Good modelling of the transverse shower width is of importance for the development of particle flow algorithms, since it affects the degree of overlap between showers, and therefore the efficiency for separating them. For each hit in the ECAL, we determine the transverse distance between the centre of the pad and the shower barycentre in $x$ and $y$. By histogramming this radial distance, we form the transverse shower profile. We weight the hits by their energy ${ }^{5}$, to emphasise the flow of energy in the shower. This also has the benefit of minimising possible residual effects of noisy cells.

In figure 8 we show these transverse energy profiles for four typical energies, compared with the simulation based on the QGSP_BERT physics list. The entries in these plots are weighted by energy, and we want to focus here on the shape rather than the normalisation, which is essentially governed by the differences in energy response which were already considered in figure 77. Therefore in figure 8 the distributions are normalised to unity, in order to facilitate the comparison of their shapes. We see that the data at $8 \mathrm{GeV}$ are quite well modelled, while at higher energies the data tend to lie below the simulation at small radii, and above at higher radii; in other words the simulation underestimates the width of that part of the shower which is contained in the ECAL.

In order conveniently to compare all models and energies, in figure 9 we show the mean energy-weighted shower radius (i.e. the mean values of distributions such as figure 8 ) as a function of energy. The observed shower becomes narrower with increasing energy, both in data and in all of the models. All of the physics lists underestimate the shower width at almost all energies, typically by around $10 \%$. The models FTF_BIC and especially FTFP_BERT tend to lie closest to the data, especially at intermediate energies. ${ }^{6}$

\footnotetext{
${ }^{5}$ In this case we do not apply different weighting factors to each stack.

${ }^{6}$ For comparison, the mean radius of electron-induced showers, selected using the methods of ref. 帄, shows agree-
} 

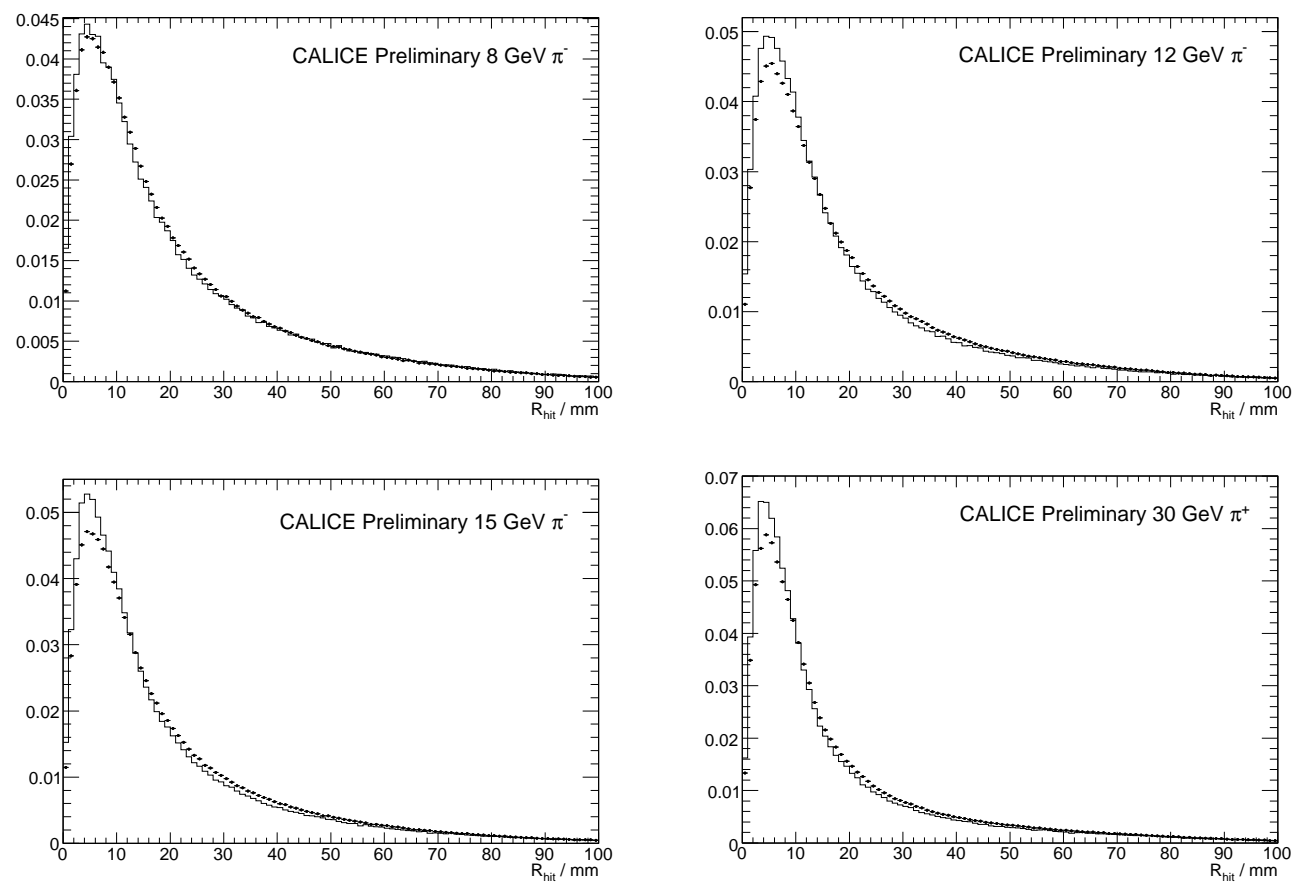

Figure 8. Radial distribution of hits (energy weighted) for data at four typical energies (points with errors) compared with Monte Carlo (solid histograms) using the QGSP_BERT physics list. The distributions are normalised to unity.
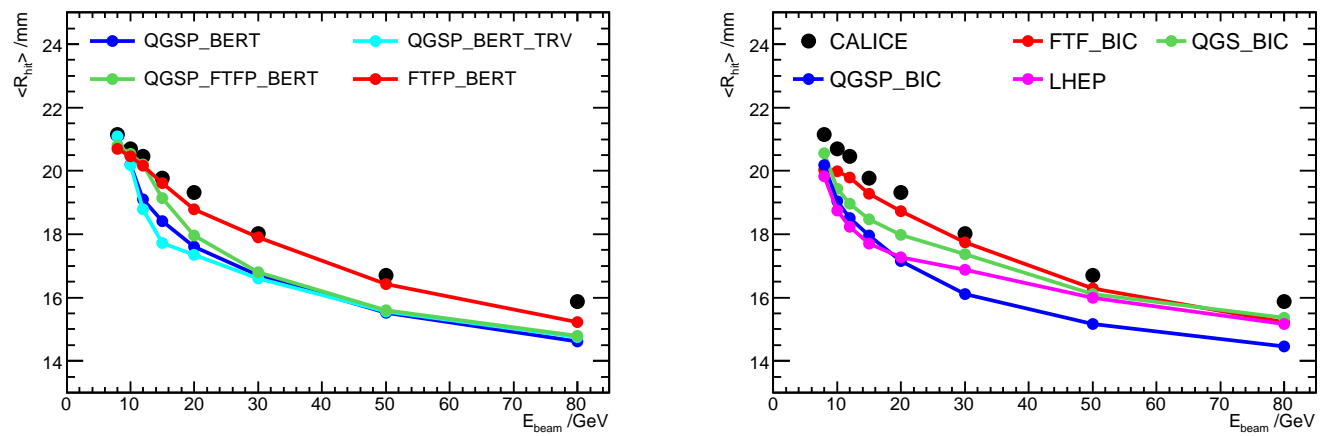

Figure 9. Mean energy-weighted shower radius in the ECAL as a function of beam energy. The data are compared with the predictions of simulations using different GEANT 4 physics lists.

Of course, the mean shower radius is only one measure of the transverse shower profile. In figure 10 we focus on the tails of the showers by plotting the radii needed to contain $90 \%$ or 95\% of the observed ECAL shower energy. As before, we find that most of the models tend to underestimate the data, and none of them gives a really satisfactory description. Again, the most successful physics lists are clearly FTFP_BERT and FTF_BIC. It should be stressed that these observations refer only to that part of the hadronic shower which is detected in the ECAL, i.e.

ment between data and simulation at the $\sim 1-2 \%$ or $0.1-0.2 \mathrm{~mm}$ level. 

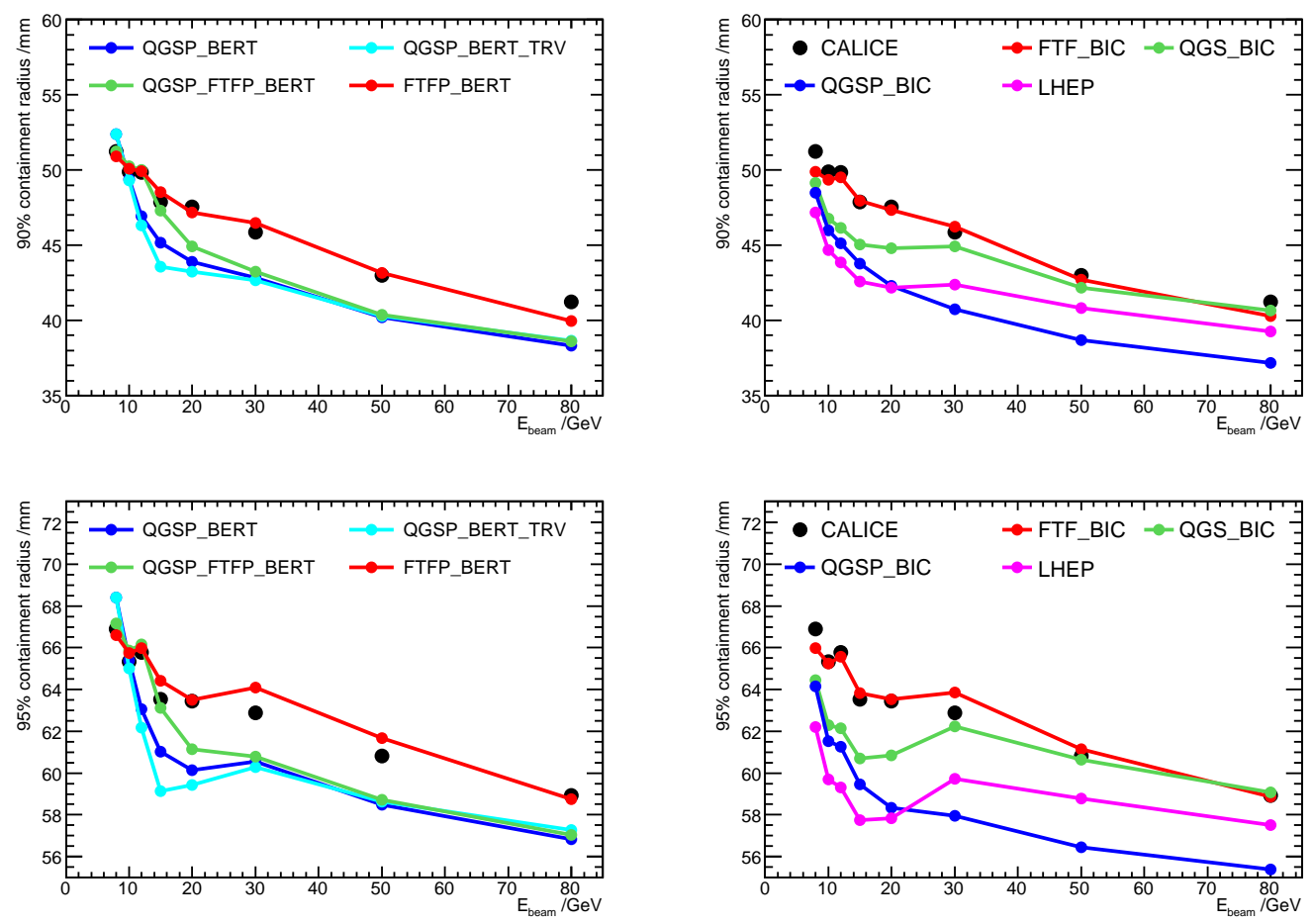

Figure 10. Radii required to contain 90\% (upper two plots) or 95\% (lower two plots) of the energy seen in the ECAL, as a function of beam energy. Data are compared with simulation for various physics lists.

roughly the first interaction length - the transverse tails are not fully sampled, and nor is most of the longitudinal tail of the shower.

\subsection{Longitudinal distribution of interaction point}

The fine granularity of the CALICE ECAL makes it possible to identify the point at which the incident pion makes its primary interaction, and this will be used in some of the results shown below. In general one expects to see a track of MIP-like hits until the interaction point, followed by some multiparticle shower structure thereafter. The algorithm used for the present analysis has the merit of simplicity. Firstly, the energy in each layer is computed, after excluding isolated hits (those with no neighbours in adjacent cells in the same layer). The interaction layer is defined as the first layer containing at least 10 MIPs of energy, provided that at least two of the following three layers also show energy greater than 10 MIPs. In simulated events, the true interaction point is stored using information about the true activity in the Monte Carlo. In figure 11 we show the correlation between the true interaction layer and that found by our algorithm, for a typical energy and Monte Carlo physics list. The correct layer is identified within \pm 1 in $\sim 70 \%$ and within \pm 2 in almost $90 \%$ of events. The mean of the difference between the true and reconstructed layer lies within \pm 1 layer for all physics lists and energies studied here.

In figure 12 we show the distribution of the reconstructed interaction layer for $30 \mathrm{GeV}$ data compared with a typical Monte Carlo run. The alternation between odd and even layers reflects the additional material associated with PCBs and the carbon-epoxy support structure; passing from an 

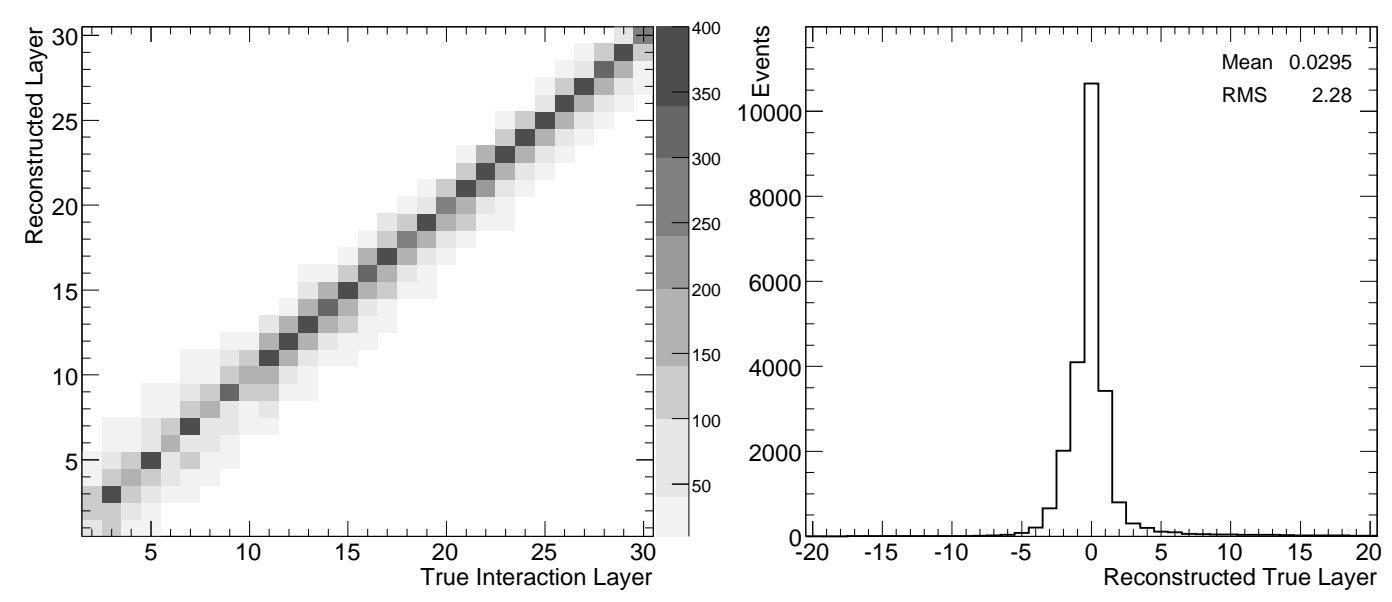

Figure 11. Left: comparison between reconstruction and truth for the layer identified as the interaction layer by the algorithm described in the text. This example corresponds to a $20 \mathrm{GeV} \pi^{-}$beam simulation, using the QGSP_BERT physics list. The grey scale indicates the number of events in each bin, where bins containing fewer than 10 events (amongst a total of more than 23000) have been suppressed for the sake of clarity. Right: distribution of (reconstructed - true) layer for this sample.

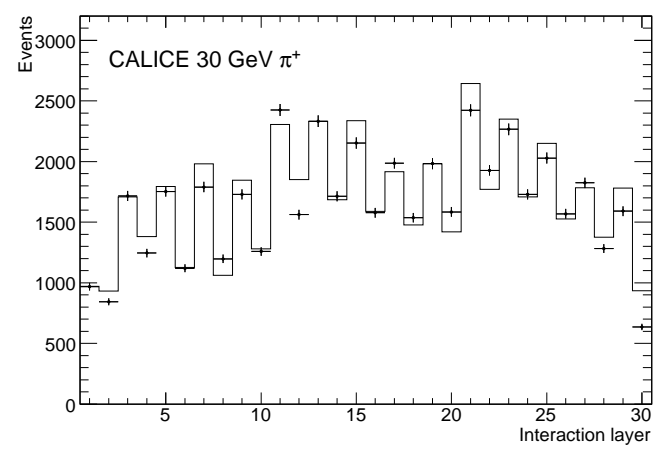

Figure 12. Distribution of the reconstructed interaction layer in the ECAL for $30 \mathrm{GeV}$ data (points), compared with Monte Carlo predictions using the QGSP_BERT physics list (solid histogram).

odd-numbered layer to an even layer, a particle will encounter just a tungsten plate, while going from an even to an odd layer it will also pass through two PCBs and part of the mechanical support structure, adding an additional $\sim 0.008 \lambda_{\text {int. }}$. The attenuation of the beam through each stack is clearly seen, becoming stronger in later stacks as the tungsten thickness increases. The upward steps at layers 10 and 20 result from the increase of thickness of the tungsten plates at these points. The lower values in layers 1 and 2 are a by-product of the cut designed to remove upstream interactions. These features are all seen in the simulation, and are generally well modelled. The following analysis concentrates on interactions in the first stack, for which the agreement is particularly good. This distribution is similarly well described at all energies by all physics lists, which suggests that there is no significant problem with the high energy cross-sections on tungsten in this version of GEANT 4. This is therefore not a directly useful distribution for discriminating between models. 


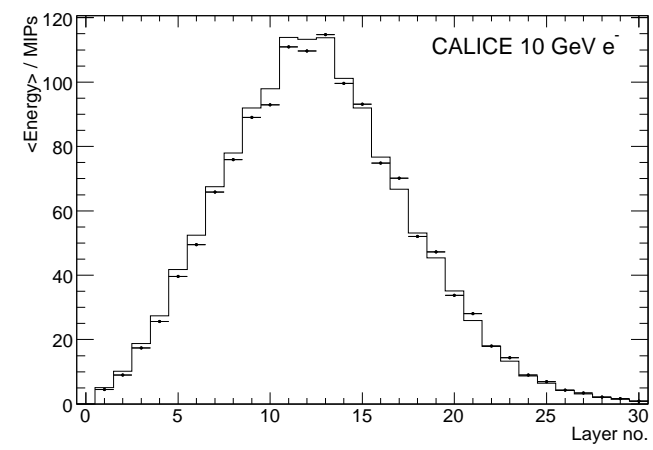

Figure 13. Energy per layer in the ECAL for $10 \mathrm{GeV}$ electron data (points), compared with Monte Carlo predictions (solid histogram).

\subsection{Longitudinal energy profile}

It is of interest to study the longitudinal development of the showers. In the case of electrons, we find that the mean depth of electron-induced showers, selected using the methods of ref. [7], shows good agreement between data and simulation, to better than $0.1 X_{0}$ (corresponding to less than $25 \%$ of a $1.4 \mathrm{~mm}$ tungsten layer), and the standard deviation is modelled to better than $0.03 X_{0}$. A typical comparison is shown in figure 13. We therefore believe that the material content of the ECAL is well modelled. The case of hadronic showers is, however, complicated by the different points in the ECAL at which the shower is initiated. The observed longitudinal distribution of energy in the ECAL is the convolution of the intrinsic shape of showers with the distribution of shower starting points. In order to have some sensitivity to the composition of the shower in terms of different particle species, we would like to measure the shower profile with respect to the primary interaction point.

In Sect. 6.3 we explained our algorithm for identifying the layer closest to the interaction point, and showed that it was reasonably reliable and well modelled. We now proceed to compute the shower profile in terms of layers after the interaction layer. This is made more complicated by the different sampling fractions in the three ECAL stacks. We circumvent this by the following expedient. For hits in the first stack (tungsten thickness $1.4 \mathrm{~mm}$ ), we simply use the measured energy in each layer. In the second stack (tungsten thickness $2.8 \mathrm{~mm}$ ), in addition to the energy measured in each layer, we introduce an additional fictitious pseudolayer midway between each physical layer, whose energy is estimated by linear interpolation between the layers on either side. Similarly, in the third stack (tungsten thickness $4.2 \mathrm{~mm}$ ) we introduce two pseudolayers between each physical layer. In this way, the energy deposition is estimated in 60 layers each separated by an effective separation of $1.4 \mathrm{~mm}$ of tungsten. The longitudinal shower profile (i.e. the mean energy, in MIPs, per layer) is then trivially computed starting at the interaction layer measuring the depth in units of these effective $1.4 \mathrm{~mm}$ layers. This calculation will lead to some non-trivial correlations between neighbouring bins in the longitudinal profile. It also neglects differences in the material between successive samplings caused by other passive materials such as PCBs, though this effect will be averaged out by combining different interaction layers. Furthermore, both these features will also be present in the simulation. For this study, we restrict the interaction layer to lie 
in the first stack, so that at least 50 effective layers are available for observation the shower profile, corresponding to $\sim 20 X_{0}$ or $0.8 \lambda_{\text {int. }}$.

In the case of the GEANT 4/Mokka simulation, it is possible to store a breakdown of the fractions of the energy of each hit contributed by various particle species. In this way, one can break down the longitudinal shower profile into its constituent parts. We choose to separate the energies deposited by electrons, positrons, "mesons" (charged pions, kaons and muons), protons (including antiprotons) and "others" (e.g. deuterons, $\alpha$-particles, hyperons).

In figure 14 we show, for $12 \mathrm{GeV} \pi^{-}$, the longitudinal energy profiles data compared with simulations using different physics lists. In each case, we also show the simulation broken down into each of the above categories. The "mesons" show a smooth slowly falling shape, while the electrons and positrons show the characteristic rise and fall of an electromagnetic shower, peaking after $\sim 10-15$ layers $\left(\sim 4-6 X_{0}\right)$. The electrons, however, also exhibit a long tail; this is because part of the ionisation energy loss of other particles is simulated in the form of discrete knockon electrons, and therefore is included in the electron contribution. The protons, and the small contribution from "others", seem to exhibit two components - a sharp peak in the first few layers which, because of its short range, can be attributed to low energy nuclear break-up fragments, and a long range tail similar to the mesons. We note that there are significant differences between the models, most conspicuously in the short range proton component. The comparison with data demonstrates that our calorimeter has sufficient longitudinal granularity to be able to offer some discrimination between these components.

The main features of the data distribution in figure 14 may be summarised as follows:

- A small peak is seen in the first few layers, which we attribute to nuclear fragments, mainly protons. As noted above, the models exhibit considerable variation in their predictions in this region, associated with differences in their proton yields. None agrees well with data, with the FTF-based physics lists overestimating the data, and the others undershooting.

- A broad peak occurs around layer 10, which we ascribe to the electromagnetic component. This is tolerably well modelled by all physics lists, with some variations in normalisation.

- A long tail follows, which appears to be generally well modelled.

In figure 15 we show similar distributions for two physics lists, FTFP_BERT and QGSP_BERT, at four typical energies. Clearly both models have imperfections, but on balance FTFP_BERT is probably the more successful.

In order to quantify these observations, and extend them to all energies and models, we show in figure 16 ratios of simulation to data as a function of energy for three regions of the longitudinal profile: layers 1-3 (where the contribution from nuclear breakup dominates), layers 5-20 (dominated by the showering of electrons and photons) and layers 30-50 (the tail, dominated by penetrating hadrons). This makes it clear that the greatest differences between the models occur in the first few layers, where discrepancies up to $\sim 20 \%$ are observed. The FTFP_BERT and FTF_BIC models lie consistently above the data, while LHEP, QGSP_BIC and QGS_BIC lie consistently below. The other physics lists make a transition between the two regimes, as their model content changes with energy. In the electromagnetic-dominated region, the most obvious outlier is LHEP; the other models all agree with data within $\sim 15 \%$, with the FTFP_BERT and FTF_BIC models 

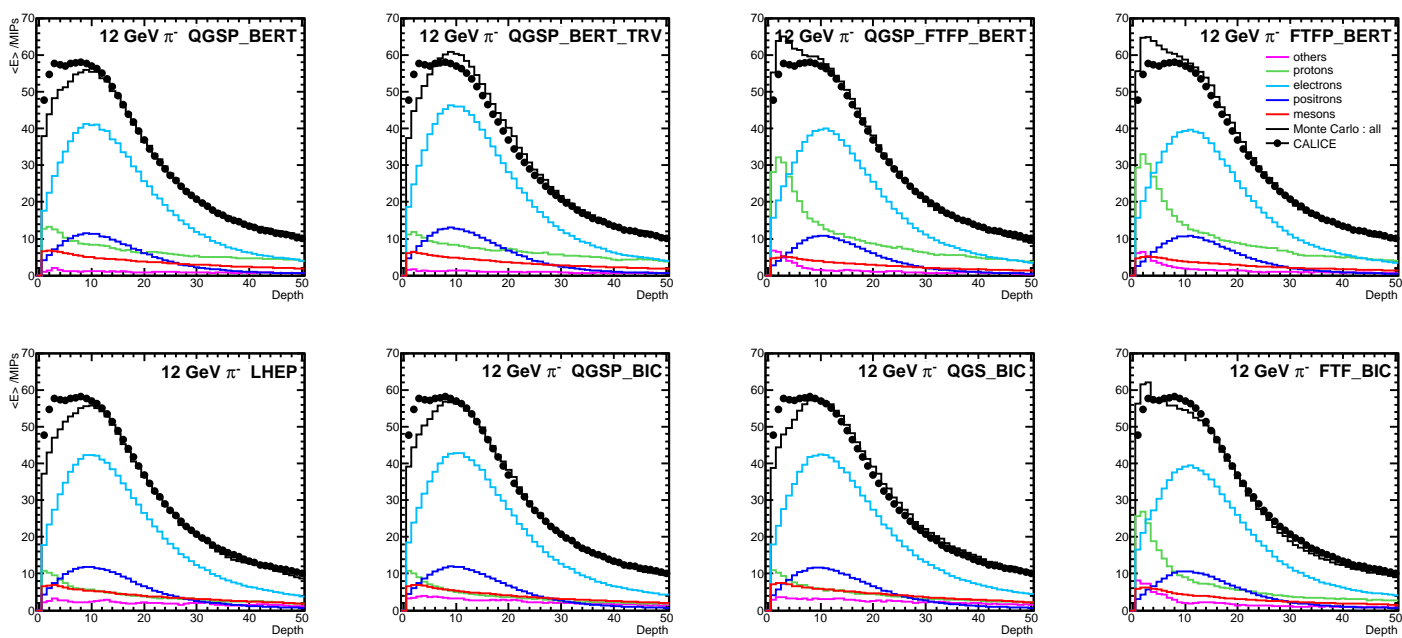

Figure 14. Longitudinal energy profiles for $12 \mathrm{GeV} \pi^{-}$data (shown as points), compared with simulations using different physics lists. The mean energy in MIPs is plotted against the depth after the initial interaction, in units of effective $1.4 \mathrm{~mm}$ tungsten layers. The total depth shown corresponds to $\sim 20 X_{0}$ or $0.8 \lambda_{\text {int. }}$. The breakdown of the Monte Carlo into the energy deposited by different particle categories is also indicated.
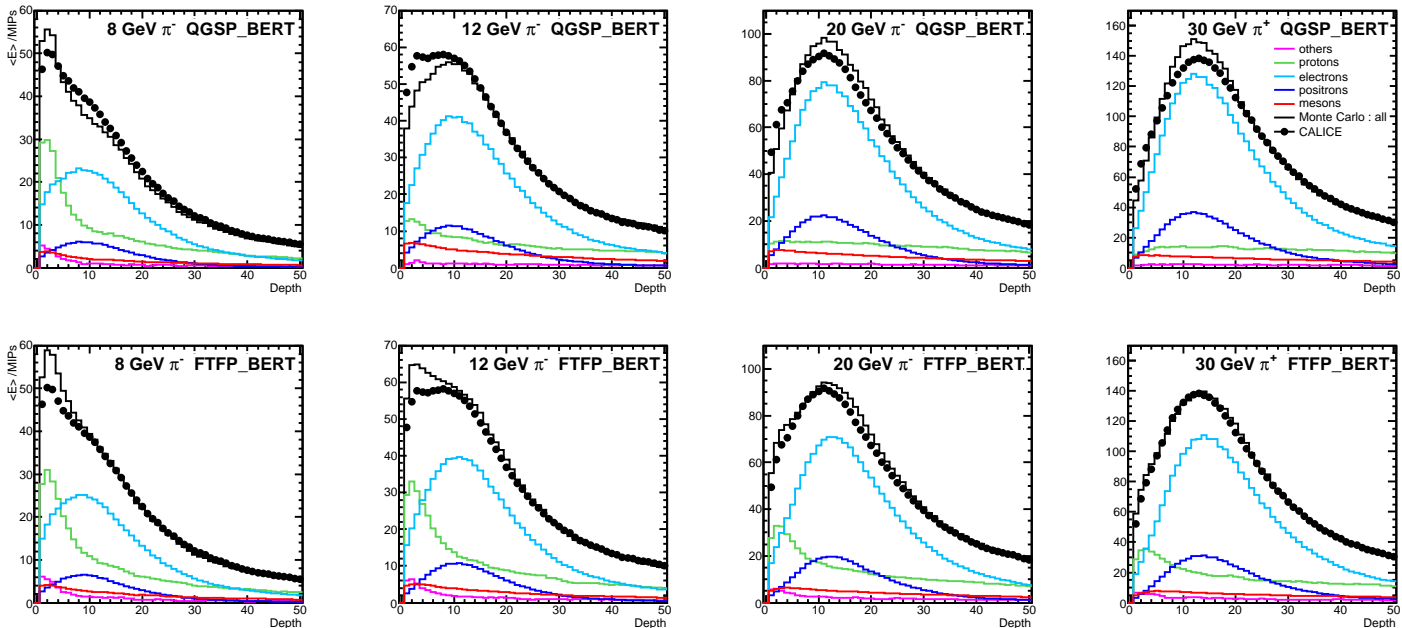

Figure 15. Longitudinal energy profiles for data (shown as points) compared with simulations using two physics lists, QGSP_BERT and FTFP_BERT, at four typical energies. The breakdown of the Monte Carlo into the energy deposited by different particle categories is also indicated.

giving the best description. In the tails, most models lie within $\sim 10 \%$ of data; LHEP is consistently low, as is FTF_BIC at lower energies.

On balance, it appears that the FTFP_BERT physics list, while not perfect, gives the best overall description of the longitudinal development of these showers. We emphasise, however, that this remark refers only to the early part of the shower which is developed in the ECAL; we are not sensitive to the later parts of the shower. 


\subsection{Physics lists under development}

There are several physics lists in GEANT 4.9.3 which use the CHIPS model [16] alone or in conjunction with other models. This model has undergone substantial development recently, and is now capable of modelling all parts of the hadronic interaction process. We made a number of studies of these physics lists using a $\beta$-test version 4.9.3.b01 of GEANT. The results were very encouraging, and one of the physics lists tested, QGSC_CHIPS, gave a better description of our data than any of the physics lists discussed here. However, the CHIP S model is still being tuned, and in the released version of GEANT 4.9.3, the CHIPS-based models are less successful when confronted with our data. It would be premature to show results and draw conclusions while development is ongoing, but the CHIPS-based models seem to be an interesting and promising new avenue, and it is clear that our data have the power to discriminate between tunings of such models.

\section{Summary}

We have studied showers induced by charged pions in the CALICE silicon-tungsten ECAL. The calorimeter has high transverse and longitudinal granularity, which allows us to study the early part of the shower development in unprecedented detail. Using pions in the energy range 8-80 GeV, we have compared the data with GEANT 4 simulations using eight different physics lists. Several observables were examined - the total energy deposited in the ECAL, the distribution of interaction points, the transverse shower energy profile, and the longitudinal profile with respect to the interaction point. The most sensitive observables seem to be the shower profiles. The data tend to show a greater shower width than the simulation for most energies and physics lists. The closest description of the transverse profiles is provided by the FTFP_BERT and FTF_BIC physics lists.

The longitudinal profile seems the most interesting observable, because the three main components of the shower induced by the primary interaction (energetic hadrons, photons and low energy nuclear fragments) can be, to some extent, distinguished through their different rates of shower development. An ECAL using tungsten is particularly useful in this regard, because of its small ratio of $X_{0} / \lambda_{\text {int. }}$, which amplifies the differences in shower development between various components of the shower. It would be naïve to expect any of the physics lists to give a perfect description of the data, but it seems clear from our study that the LHEP physics list has serious deficiencies, and that, in the framework of the current version 4.9.3 of GEANT 4, FTFP_BERT list is the most successful. It is to be hoped that these observations will provide useful guidance to the GEANT 4 developers.

\section{Acknowledgements}

We would like to thank the technicians and the engineers who contributed to the design and construction of the prototypes. We also gratefully acknowledge the DESY and CERN managements for their support and hospitality, and their accelerator staff for the reliable and efficient beam operation. We would like to thank the HEP group of the University of Tsukuba for the loan of drift chambers for the DESY test-beam. The authors would like to thank the RIMST (Zelenograd) group for their help and sensors manufacturing. This work was supported by the Bundesministerium für Bildung und Forschung, Germany; by the DFG cluster of excellence "Origin and Structure of the Universe"; by the Helmholtz-Nachwuchsgruppen grant VH-NG-206; by the BMBF, 

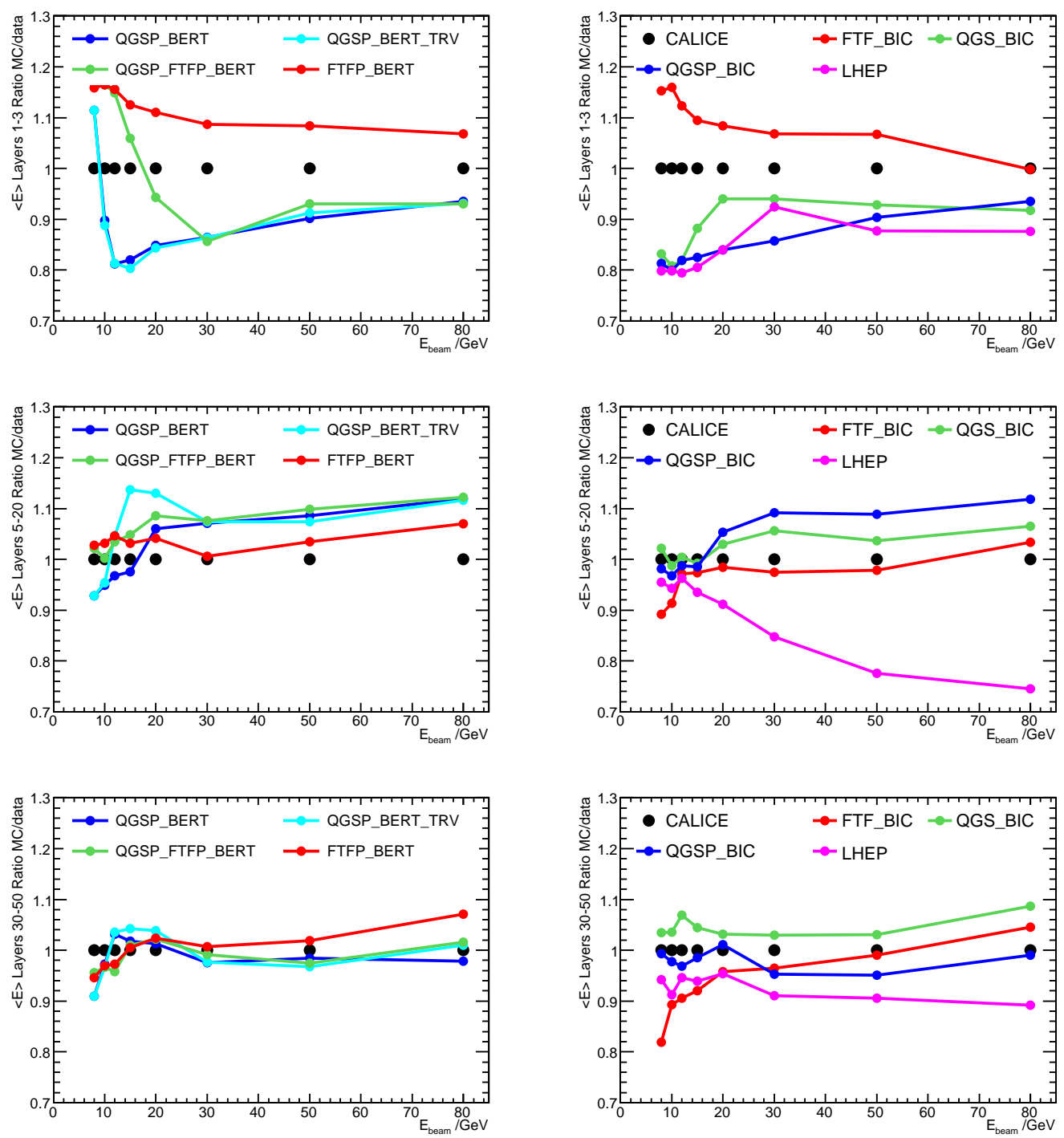

Figure 16. Ratio of simulation to data for three different regions of the longitudinal energy profile: (top pair of plots) layers 1-3, dominated by nuclear breakup; (centre pair) layers 5-20, dominated by electromagnetic showers; and (bottom pair) layers 30-50, dominated by penetrating hadrons.

grant numbers 05HS6VH1 and 05HS6GU1; by the Alexander von Humboldt Foundation (Research Award IV, RUS1066839 GSA); by joint Helmholtz Foundation and RFBR grant HRJRG002, Russian Agency for Atomic Energy, ISTC grant 3090; by Russian Grants SS-1329.2008.2 and RFBR0402/17307a and by the Russian Ministry of Education and Science; by CICYT,Spain; by CRI(MST) of MOST/KOSEF in Korea; by the US Department of Energy and the US National Science Foundation; by the Ministry of Education, Youth and Sports of the Czech Republic under the projects AV0 Z3407391, AV0 Z10100502, LC527, LA09042 and by the Grant Agency of the Czech Republic under the project 202/05/0653; and by the Science and Technology Facilities Council, UK. 


\section{References}

[1] J.-C. Brient and H. Videau arXiv:hep-ex/0202004v1 (2002);

V.L. Morgunov, in $10^{\text {th }}$ International Conference on Calorimetry in High Energy Physics (CALOR 2002), Pasadena, CA, Mar. 2002.

M.A. Thomson, Nucl. Instrum. and Methods A611 (2009) 25-40.

[2] J. Repond et al., JINST 3 (2008) P08001.

[3] http://ab-div-atb-ea.web.cern.ch/ab-div-atb-ea/BeamsAndAreas/H6

[4] C. Adloff et al., arXiv: 1003.2662 , to be published in JINST.

[5] S.Agostinelli et al., Nucl. Instrum. and Methods A506 (2003) 250;

J.Allison et al., IEEE Transactions on Nuclear Science 53 No. 1 (2006) 270.

[6] Mokka home page, http://polzope.in2p3.fr:8081/MOKKA;

P. Mora de Freitas and H. Videau, "Detector simulation with MOKKA / GEANT4: Present and future”, International Workshop on Linear Colliders (LCWS 2002), Jeju Island, Korea, 26-30 Aug 2002.

[7] C. Adloff et al., Nucl. Instrum. and Methods A608 (2009) 372.

[8] GEANT4 Physics Reference Manual,

http://geant 4 web.cern.ch/geant 4/UserDocumentation/

/UsersGuides/PhysicsReferenceManual/fo/PhysicsReferenceManual.pdf

[9] H. Fesefeldt Simulation of Hadronic Showers, Physics and Applications. Technical Report PITHA 85-02, (1985).

[10] M. P. Guthrie, R. G. Alsmiller, H. W. Bertini, Nucl. Instrum. and Methods A66 (1968) 29;

A. Heikkinen, N. Stepanov and J.-P. Wellisch, Proc. Computing in High Energy and Nuclear Physics, vol. MOMT008, La Jolla, CA, 2003.

[11] B. Andersson et al., Nucl. Phys. B281 (1987) 289;

B. Nillson-Almquist and E. Stenlund, Comp. Phys. Commun. 43 (1987) 387.

[12] G. Folger, J.-P. Wellisch, arXiv:nucl-th/0306007 (2003);

N. S. Amelin, et al.,Phys.Rev.Lett. 67 (1991) 1523;

L. V. Bravina, et al., Phys.Rev.Lett. 344 (1995) 49.

[13] V. Lara, J.-P. Wellisch, Proceedings of the CHEP 2000 Conference, Padova, Italy, February 2000

[14] G. Folger, V. N. Ivanchenko and J.-P. Wellisch Eur. Phys. J. A21 (2004) 404

[15] See, for example, presentations by S. Banerjee and M. Asai at the $14^{\text {th }}$ GEANT4 Users Workshop, October 2009; http://indico.cern.ch/conferenceDisplay.py? confId=44566

[16] P. V. Degtyarenko, M. V. Kossov, H.-P. Wellisch, Eur. Phys. J. A8 (2000) 217; Eur. Phys. J. A9 (2000) 411; Eur. Phys. J. A9 (2000) 421. 


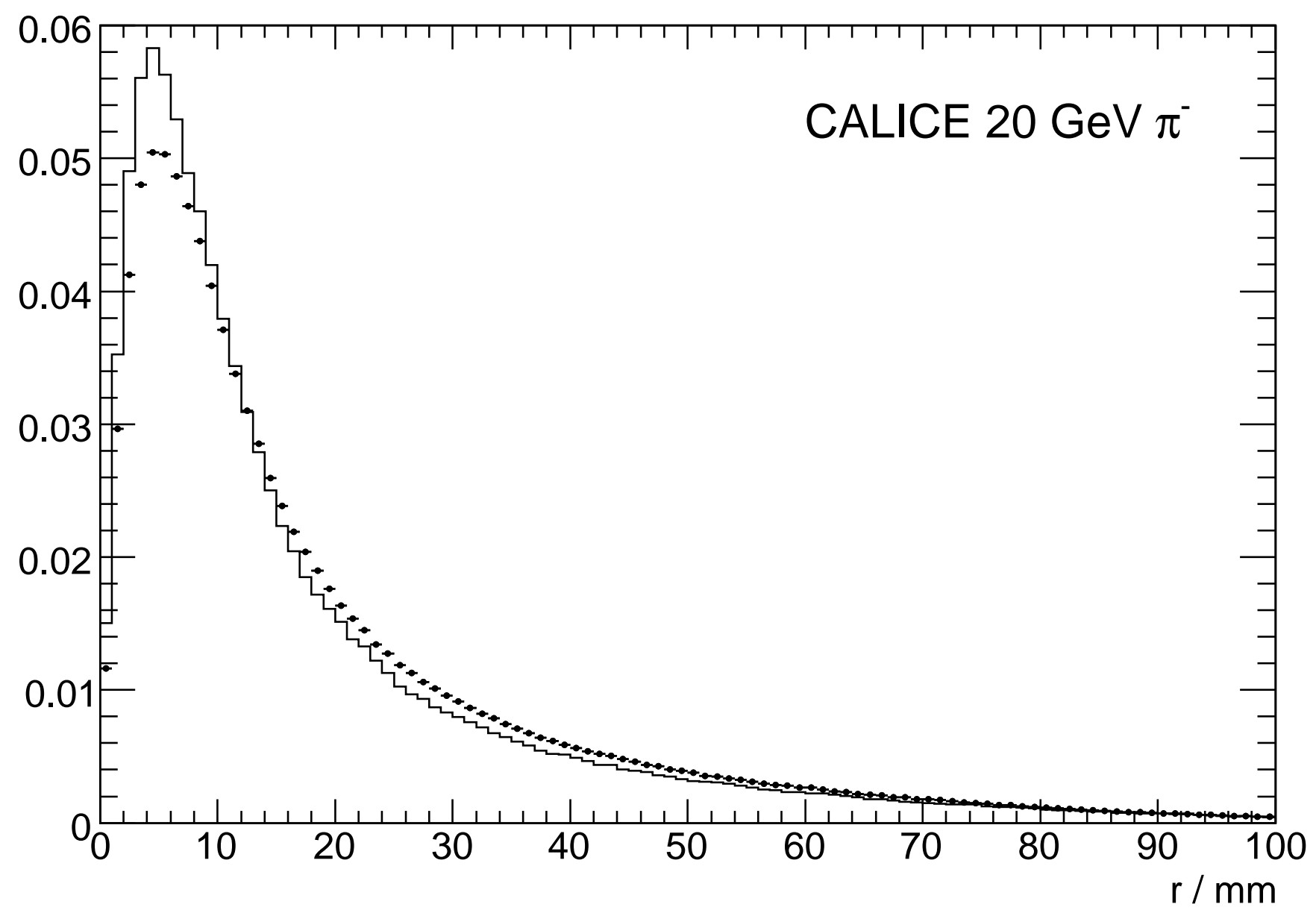




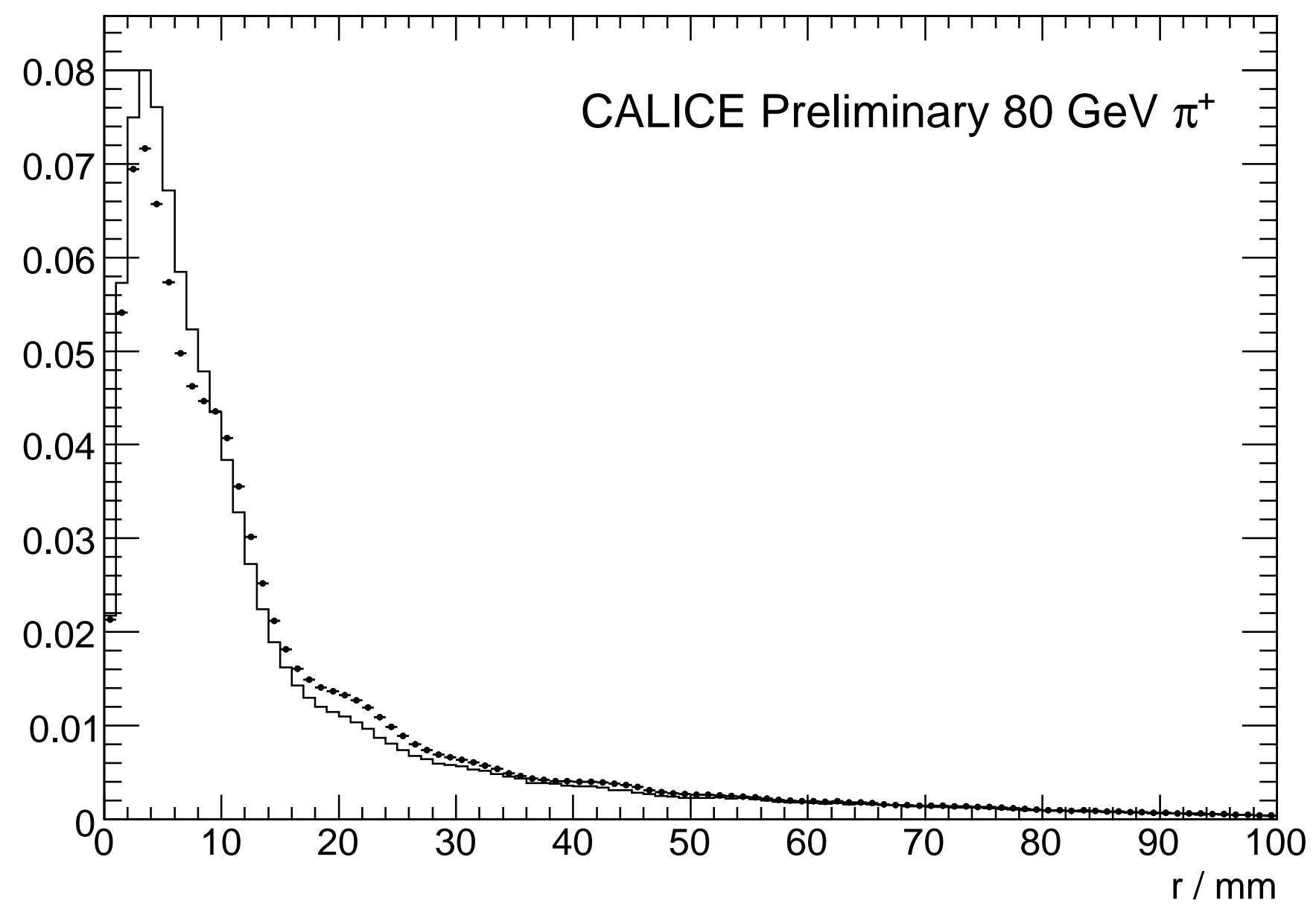

